\title{
Aging and Chronic Respiratory Diseases: Novel Mechanisms
}

\author{
Jacobo Sellares, $\mathrm{PhD}^{1,2,4}$ and Mauricio Rojas, $\mathrm{MD}^{1,2,3}$
}

${ }^{1}$ Division of Pulmonary, Allergy, and Critical Care Medicine, Department of Medicine, and ${ }^{2}$ The Dorothy P. and Richard P. Simmons Center for Interstitial Lung Disease, University of Pittsburgh Medical Center, Pittsburgh, PA, USA; ${ }^{3}$ McGowan Institute for Regenerative Medicine, University of Pittsburgh School of Medicine, Pittsburgh, PA, USA; ${ }^{4}$ Servei de Pneumologia, Hospital Clinic, IDIBAPS, Universitat de Barcelona, Barcelona, Spain

\section{ABSTRACT}

Aging is a progressive loss of physiological integrity leading to impaired function and increased vulnerability to death. Research into the mechanisms of aging could contribute to a better understanding of many chronic diseases. Chronic obstructive pulmonary disease (COPD) and idiopathic pulmonary fibrosis (IPF) are chronic respiratory diseases associated with aging due to the fact that "accelerated" aging of the lung has been proposed as an important mechanism in both diseases. Many of the hallmarks that have been described in aging are also present in chronic obstructive pulmonary disease and idiopathic pulmonary fibrosis. Telomere attrition, loss of proteostasis, mitochondrial dysfunction, cellular senescence, and stem cell exhaustion are all mechanisms of aging present in both diseases. In this review we will examine the underlying mechanisms that link aging with idiopathic pulmonary fibrosis and chronic obstructive pulmonary disease. (BRN Rev. 2017;3:18-29) Corresponding author: Mauricio Rojas, rojasm@upmc.edu

Key words: Aging. Cellular senescence. COPD. IPF. 


\section{INTRODUCTION}

Aging is a process that affects all human beings physically, emotionally, and psychologically. In recent years, different publications have addressed the process of aging from a scientific perspective. In recent decades, life expectancy (average total number of years that a human expects to live) has been progressively increasing, especially in developed countries $^{1}$. It is estimated that the 65-yearsold and over populations will increase from 41.4 million in 2011 to 79.7 million by 2040 in the USA ${ }^{2}$.

Many different chronic diseases that represent a serious burden for the medical community are associated with the process of aging. In the case of lung diseases, COPD and IPF are described as diseases associated with aging. In this paper, we review the biology of aging and its relationship with the pathogenesis of IPF and COPD.

\section{CELLULAR AND MOLECULAR PROCESSES ASSOCIATED WITH AGING AND CHRONIC RESPIRATORY DISEASES}

From a biological point of view, aging is a progressive loss of physiological integrity, leading to impaired function and increased vulnerability to death ${ }^{3}$. A group of experts have described different hallmarks that contribute to the process of aging: genomic instability, telomere attrition, epigenetic alterations, loss of proteostasis, deregulated nutrient sensing, mitochondrial dysfunction, cellular senescence, stem cell exhaustion, and altered intercellular communication ${ }^{3}$ (Table 1). The

\begin{tabular}{|c|c|}
\hline Hallmarks of aging ${ }^{3}$ & $\begin{array}{l}\text { Pillars of aging } \\
\text { (GeroScience group) }^{4}\end{array}$ \\
\hline $\begin{array}{l}\text { Epigenetic alterations } \\
\text { Genomic instability } \\
\text { Telomere attrition }\end{array}$ & Epigenetics \\
\hline \multirow{2}{*}{$\begin{array}{l}\text { Loss of proteostasis } \\
\text { Deregulated nutrient sensing } \\
\text { Mitochondrial dysfunction }\end{array}$} & Proteostasis \\
\hline & Macromolecular damage \\
\hline \multirow{2}{*}{$\begin{array}{l}\text { Cellular senescence } \\
\text { Stem cell exhaustion } \\
\text { Altered intercellular communication }\end{array}$} & Stem cells and regeneration \\
\hline & $\begin{array}{l}\text { Inflammation } \\
\text { Adaptation to stress } \\
\text { Metabolism }\end{array}$ \\
\hline
\end{tabular}

authors proposed that each hallmark should ideally fulfil the following criteria: (i) it should manifest itself during normal aging; (ii) its experimental aggravation should accelerate aging; and (iii) its experimental amelioration should retard the normal aging process and hence extend the healthy lifespan. Most of these mechanisms could interact and contribute globally to the process of aging ${ }^{3}$. Genomic instability, telomere attrition, epigenetic alterations, and loss of proteostasis are the primary hallmarks that cause the initial damage. After this initial damage, mitochondrial dysfunction, cellular senescence, and deregulated nutrient sensing develop to mitigate the damage. These processes could be deleterious when prolonged during the lifespan. Finally, integrative hallmarks such as stem cell exhaustion and altered intercellular communication are the final results of the initial injury and are directly responsible for the functional damage associated with aging (Fig. 1).

More recently, the National Institute of Health (NIH) GeroScience Interest Group (GSIG) ${ }^{4}$ 

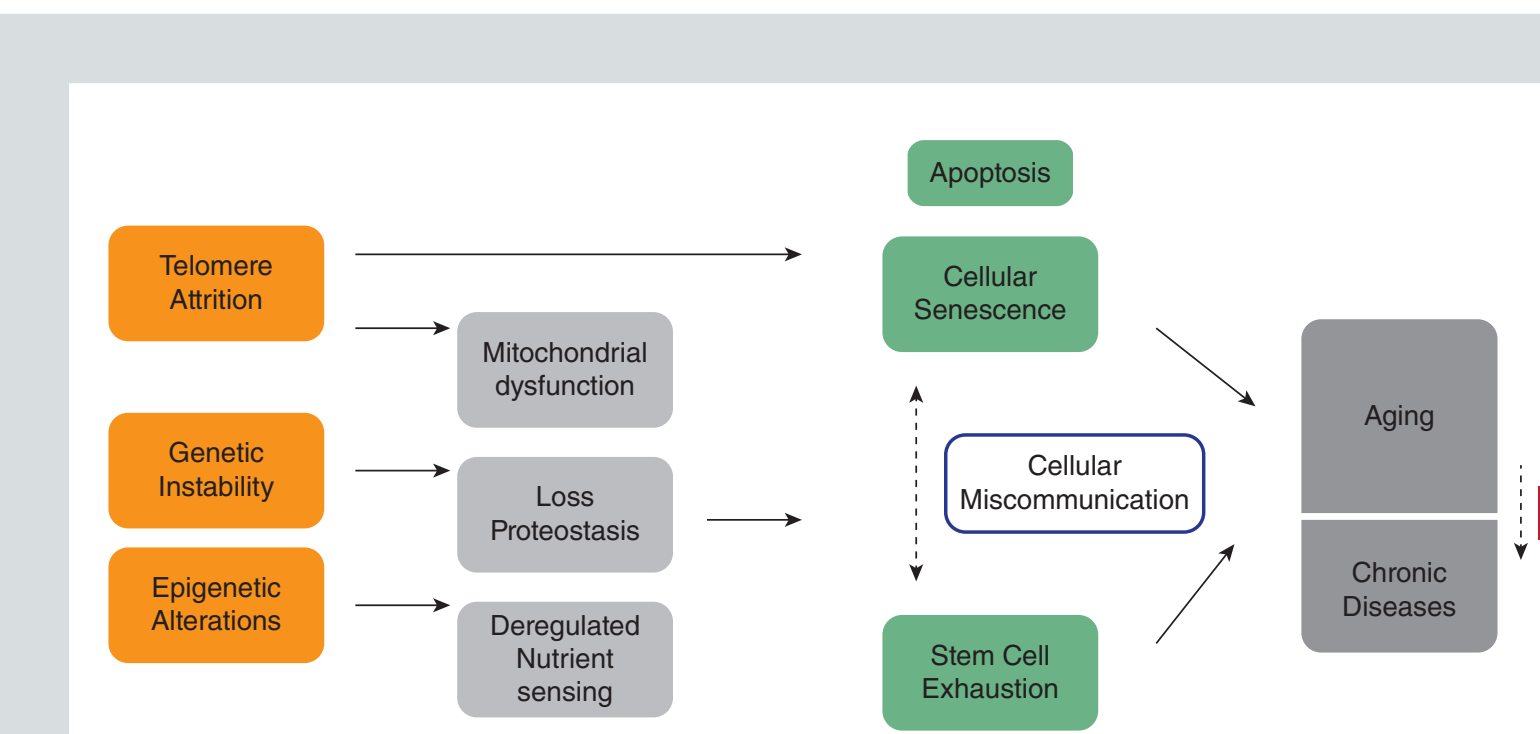

Figure 1. Mechanisms involved in chronic diseases.

has described what it considers to be the pillars of aging: macromolecular damage, epigenetics, inflammation, adaptation to stress, proteostasis, stem cells, and regeneration and metabolism. Based on these different pillars or areas, the group has proposed important goals to achieve in the coming years (Table 1). Interestingly, the authors did not define these characteristics as seven independent factors driving aging; rather, they understood they were inseparably intertwined processes, and that the interplay between these seven pillars was critical. In addition, although the themes in "pillars of aging" were more general, epigenetics, proteostasis, and stem cells coincided in both approaches.

The different characteristics of aging are involved in many chronic respiratory disorders. In fact, many authors consider COPD and IPF to be model diseases of "accelerated" aging in the lung. When the normal lung is challenged by an injury, depending on whether the response is local ${ }^{5}$ or systemic ${ }^{6}$, a different disease is developed (Fig. 2). Additionally, aging modifies the capacity of "defensive" responses in the lung. Along these lines, different therapies directly related to several hallmarks of aging could be currently applied and consequently could improve the deficient response observed during aging (Fig. 3).

COPD is a chronic disease with high morbidity and mortality. It is thought that the prevalence of the disease will continue to rise and it is expected to be the third most common cause of death worldwide by $2020^{7}$. Although smoking is the main risk factor for the development of $\mathrm{COPD}^{8}$, this disease is also considered a type of accelerated lung aging because it is uncommon before the age of 40 and its prevalence increases after 60 years of age'.

IPF is a chronic interstitial lung disease characterized by progressive dyspnoea and the 


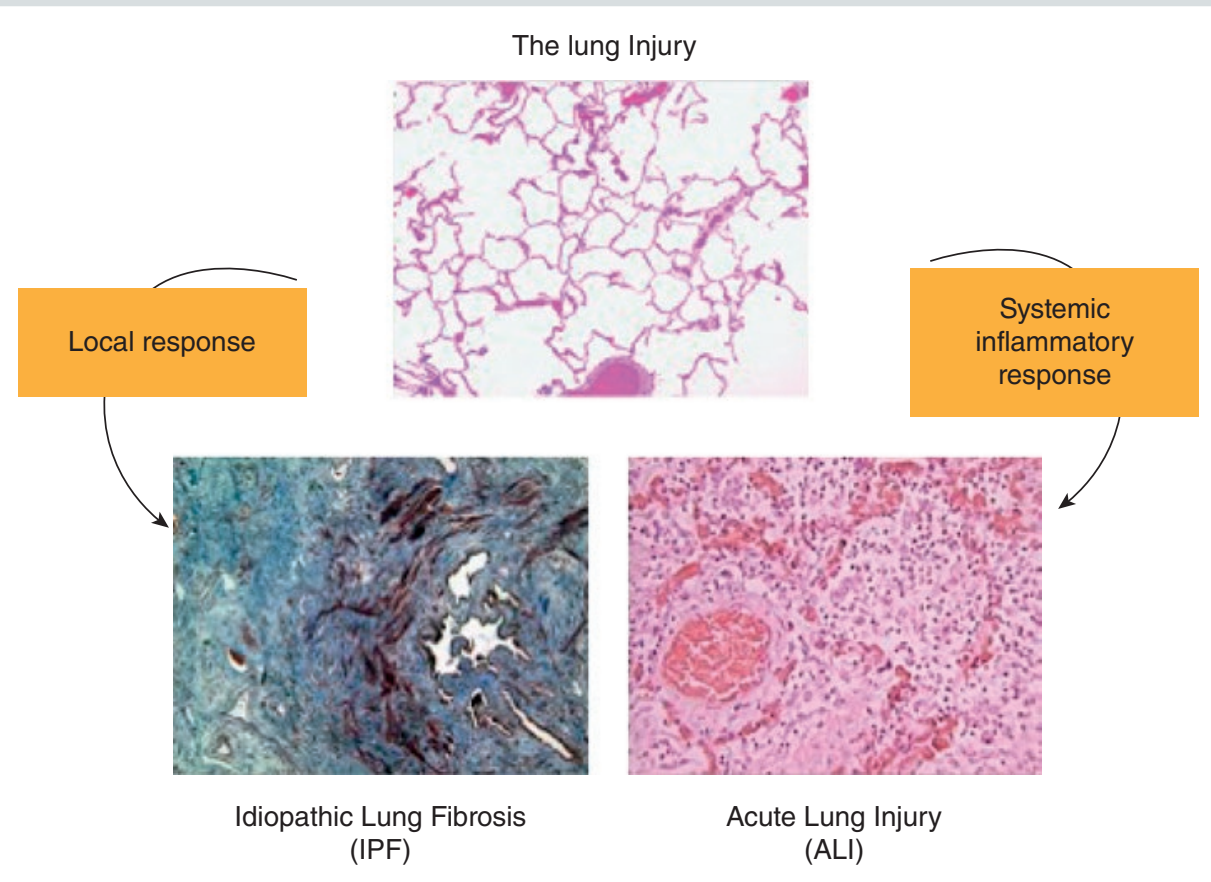

Figure 2. Different responses to lung injury.

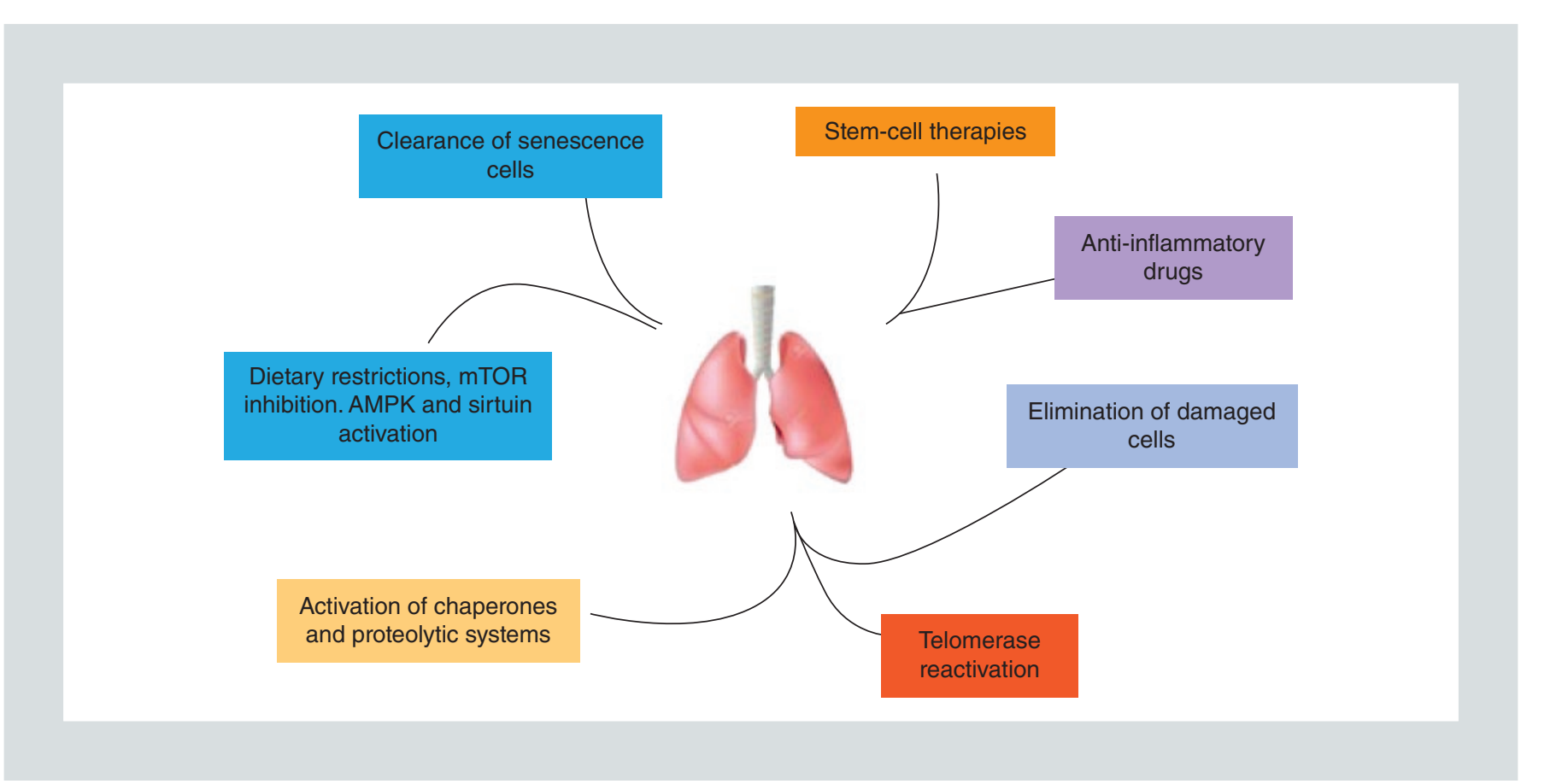

Figure 3. Interventions to modify age-related lung diseases.

AMPK: 5' AMP-activated protein kinase; mTOR: mechanistic target of rapamycin. 
irreversible loss of lung function. Its annual incidence in the USA has been estimated to be around 6.8-16.3 cases per 100,000 inhabitants $^{10}$. Its evolution is heterogeneous and may vary in form, ranging from a slow progression to accelerated forms and acute exacerbations. However, progressive deterioration is unavoidable, with a median survival of 3-5 years from the time of diagnosis ${ }^{11}$. The disease is characterized by the aberrant activation of epithelial cells, which secrete numerous mediators, resulting in expansion of the fibroblast/myofibroblast population, with the subsequent exaggerated accumulation of extracellular matrix and destruction of the lung architecture ${ }^{12}$. As a disease that typically occurs in elderly people, it has been suggested that common mechanisms could be involved in aging and IPF5.

\section{TELOMERE ATTRITION}

Telomeres are particularly susceptible to age-related deterioration. Telomere shortening is observed in normal aging ${ }^{13}$. To avoid the process of loss of deoxyribonucleic acid (DNA) in the telomeres with subsequent mitosis, a specialized DNA polymerase named "telomerase" is present in many cells. However, abnormal telomerase dysfunction and consequently accelerated telomere shortening could induce premature aging and the development of many diseases ${ }^{3}$. Telomerases have two core components: TERT, the telomerase reverse transcriptase, and $\mathrm{TR}$, the telomerase RNA that provides the template for telomere repeat addition ${ }^{14}$. Mutations in TERT and TR cause telomerase haploinsufficiency and short telomere defects, which are frequently recognized in many chronic diseases.

\section{Telomere attrition in COPD}

Telomere attrition is one of the most important hallmarks of aging. It has been demonstrated that telomerase-null mice with short telomeres develop emphysema after chronic cigarette smoke exposure ${ }^{15}$. In two human cohorts, the prevalence of TERT mutations was found in $1 \%$ of patients. In fact, in families, emphysema showed an autosomal-dominant inheritance pattern, along with pulmonary fibrosis and other telomere syndrome features, but manifested only in smokers ${ }^{16}$.

\section{Telomere attrition in idiopathic pulmonary fibrosis}

In familial pulmonary fibrosis, defined as idiopathic interstitial pneumonia in two or more first-degree relatives (parent, sibling, or offspring), telomerase mutations are the most common identifiable genetic cause of the disease ${ }^{17,18}$. Further, in sporadic IPF, telomere shortening is observed even in the absence of telomerase mutations ${ }^{19}$. The telomere length defect is seen in multiple leukocyte subsets as well as lung epithelial cells ${ }^{20}$. This observation has led to the idea that short telomere length may be a risk factor for this disease $\mathrm{e}^{21}$. In addition, a subset of IPF patients concomitantly develops cryptogenic liver cirrhosis, another telomere-mediated phenotype ${ }^{20}$. A subsequent study reported that shorter leucocyte telomere lengths were associated with lower survival rates in $\mathrm{IPF}^{22}$. Mutations in encoding polyadenylation-specific ribonuclease deadenylation nuclease (PARN) and encoding regulator of telomere elongation helicase 1 (RTEL1), genes that are related to telomere maintenance, have also been recently involved in familial pulmonary fibrosis and telomere shortening ${ }^{23}$. 


\section{MITOCHONDRIAL DYSFUNCTION}

Mitochondrial dysfunction is observed in many different chronic respiratory diseases ${ }^{24}$. With aging, the efficacy of the respiratory chain tends to be reduced, thus increasing electron leakage and reducing adenosine triphosphate (ATP) production ${ }^{25}$. Mitochondrial dysfunction is associated with an increase in reactive oxygen species (ROS) that has classically been associated with aging. However, the increase in ROS may prolong lifespan in yeast and $C$. Elegans $^{3}$. The hypothesis is that the primary effect of ROS would be the activation of compensatory homeostatic responses. As chronological age advances, cellular stress and damage increase and the levels of ROS increase in parallel in an attempt to maintain survival. Beyond a certain threshold, ROS levels eventually aggravate, rather than alleviate, the age-associated damage, which could explain the increased ROS that has been associated with age-related diseases ${ }^{3}$. Aging also could be associated with different mutations of the mitochondrial genes that may impair important proteins of the respiratory chain complex. On the other hand, chronic oxidative stress could result in mitochondrial DNA damage and abnormal oxidative phosphorylation ${ }^{26}$.

\section{Mitochondrial dysfunction in COPD}

In COPD, evidence of oxidative stress in the lungs, blood, and skeletal muscle has been observed, which could lead to mitochondrial dysfunction ${ }^{27}$. Recently ${ }^{28}$ it has been demonstrated that iron-responsive element-binding protein 2 (IRP2), a protein that regulates cellular iron homeostasis, is greater in COPD. The IRP2 increases mitochondrial iron loading and levels of cytochrome $c$ oxidase (COX), which leads to mitochondrial dysfunction and subsequent experimental COPD.

Other factors have also been described in mitochondria from COPD patients. Prohibitin-1 (PHB1), a mitochondrial protein that interacts with the nicotinamide adenine dinucleotide (NADH) dehydrogenase protein complex, and is linked to aging, is significantly downregulated in bronchial epithelial cells from patients with $\mathrm{COPD}^{29}$. Recent studies suggest that E3 ubiquitin ligase, Parkin/PARK2, and phosphatase and tensin homologue (PTEN)-induced putative protein kinase 1 (PINK1) are the main regulators in the elimination of dysfunctional mitochondria ${ }^{26}$. Expression of PINK1 is increased in COPD, suggesting excessive mitophagy responsible for necroptosis of bronchial and alveolar epithelial cells ${ }^{30,31}$. In fact, the PINK1 ${ }^{-/}$ mice are protected against cigarette smoke-induced mitochondrial dysfunction ${ }^{31}$. Additionally, in macrophages from smokers, mitophagy is impaired due to a failure in the autophagic flux $^{32}$. Mitophagy in COPD could be one of the potential explanations for the persistence of ROS and why patients with COPD have fragmented mitochondria despite smoking cessation.

\section{Mitochondrial dysfunction in idiopathic pulmonary fibrosis}

Patients with IPF also have increased markers of oxidative stress, both locally in the lungs and systemically ${ }^{33}$. Limited information is available about the role that mitochondria dysfunction could have in IPF. A recent study ${ }^{34}$ demonstrated that alveolar type II cells (AECII) in the lungs of IPF patients exhibit marked accumulation of dysmorphic and dysfunctional 
mitochondria. These mitochondrial abnormalities in AECIIs of IPF lungs were associated with upregulation of endoplasmic reticulum (ER) stress markers and were also seen in normal mice of advancing age in response to stimulation of ER stress. In addition, impaired mitochondria in IPF and aging lungs were associated with low expression of PINK1, a kinase that plays an important role in the maintenance of mitochondrial morphology and function and in the selective degradation of damaged mitochondria by mitophagy.

We have recently reported that fibroblasts isolated from IPF explanted lungs have fewer mitochondria and decreased respiration and glycolysis, leading to a lower ATP production compared to age-matched controls ${ }^{35}$.

\section{LOSS OF PROTEOSTASIS}

Proteostasis is the process of protein homeostasis, which involves mechanisms for the stabilization of correctly folded proteins (most prominently ruled by the heat shock family of proteins, HSP) and mechanisms for the degradation of proteins by the proteasome or the lysosome $e^{3}$. Molecular chaperones are central to the function of proteostasis and can be broadly grouped into the HSP70, HSP90, DNAJ/ HSP40, chaperonin/HSP60, and small HSP (sHSP) families. From these chaperones, HSP70 and HSP90 are the best studied ${ }^{36}$. With aging, proteostasis is altered ${ }^{37}$. Additionally, as a consequence of proteostasis imbalance, chronic expression of unfolded, misfolded, or aggregated proteins could contribute to the development of some age-related pathologies, such as Alzheimer's disease, Parkinson's disease, and cataracts $^{38}$. The causes of loss of proteostasis could be influenced because the chaperones implied in proteostasis are impaired or the decline of proteolytic systems with aging. Accumulation of damaged or misfolded proteins in the endoplasmic reticulum, a condition termed "ER stress", induces a compensatory cellular response termed the "unfolded protein response". When ER stress cannot be reversed, the unfolded protein response induces cell apoptosis ${ }^{39}$. On the other hand, the decline of autophagy (a function that selectively degrades and removes damaged proteins, organelles, and pathogens via lysosomes) is present in many diseases associated with aging, particularly when it is prolonged or excessive ${ }^{26}$.

\section{Loss of proteostasis in COPD}

The role of the imbalance in proteostasis has not been extensively studied in COPD. In the case of chaperones, circulating levels of HSP70 have been associated with an increased risk of $\mathrm{COPD}^{40}$. Additionally, the loss of Hsp90 expression in lymphocytes has been implicated in steroid resistance in COPD ${ }^{41}$. In relation to mechanisms involved in autophagy, scientific research is divided into evidence that demonstrates that the autophagy is excessive in $\mathrm{COPD}^{42}$, and studies showing that it could be defective ${ }^{43}$. The markers of autophagy are increased in lung peripheral tissue from patients with COPD, suggesting a possible increase in autophagy ${ }^{42}$.

\section{Loss of proteostasis in idiopathic pulmonary fibrosis}

The regulation and the maintenance of proteostasis in IPF have not been extensively 
studied, and the few studies have mainly focused on autophagy. Light chain 3-II (LC3-II) levels, a marker of autophagy, are significantly lower in lungs from patients with $\mathrm{IPF}^{44}$. In addition, aging contributes to a lower induction of autophagy on basal conditions and under starvation of IPF fibroblasts, mediated by mechanistic target of rapamycin (mTOR) pathway activation ${ }^{45}$. Interestingly, treatment with rapamycin and PP242, targeting the PI3K/AKT/mTOR signalling pathway, modifies starvation-induced autophagy and apoptosis in IPF fibroblasts ${ }^{45}$.

\section{CELLULAR SENESCENCE}

Senescence is the key hallmark of aging. Cellular senescence can be defined as a stable arrest of the cell cycle coupled to stereotyped phenotypic changes ${ }^{3}$. Telomere shortening is the main cause of cellular senescence, although non-telomeric DNA damage and depression of the inhibitors of CDK4 (INK4)/ alternative reading frame (ARF) locus could also induce senescence ${ }^{46}$. As the amount of senescent cells increases with aging, it has been thought that senescence contributes to aging. Although senescence could be a beneficial response to avoid the expansion of damaged cells, the accumulation of these cells due to increased formation of senescent cells and reduction of reparative mechanisms could lead to progressive loss of tissue functions ${ }^{3}$. In addition, senescent cells manifest changes in their secretome, which is particularly enriched in proinflammatory cytokines and matrix metalloproteinases. These changes are referred to as the "senescence-associated secretory phenotype" (SASP), which could contribute to aging ${ }^{47}$. Taking into account recent available research from our group and others, we postulate that "accelerated" cellular senescence is the main mechanism in most chronic diseases like COPD and IPF. An analysis of senescence in tissues could be carried out by examining different markers. The most widely used assay for senescence is the detection of $\beta$ galactosidase activity, which is known as senescenceassociated $\beta$-galactosidase (SA $\beta G A L$ ). This activity is based on the increased lysosomal content of senescent cells, and probably reflects the increased autophagy occurring in senescent cells together with an enlargement of the lysosomal compartment. As cellular senescence is based on stable cell cycle arrest, the absence of proliferative markers, such as Ki67 protein or 5bromodeoxyuridine (BrdU) incorporation, is an essential condition to document senescence. Other canonical senescence markers comprise the most common mediators of senescence, including p16, ARF, p53, p21, p15, and p2748.

\section{Cellular senescence in COPD}

Many studies have demonstrated the presence of cellular senescence in COPD. In emphysematous lungs, there is considerable evidence for enhanced expression of p21, p16, p19, and SA $\beta$ GAL activity ${ }^{26}$. Different cell types implicated in COPD show signs of senescence. It has been demonstrated that senescence affecting fibroblast and endothelial cells could favour the development of emphysema ${ }^{49}$. Patients with emphysema had significantly higher percentages of type II endothelial cells and were positive for $\mathrm{p} 16^{\mathrm{INK} 4 \mathrm{a}}$ and

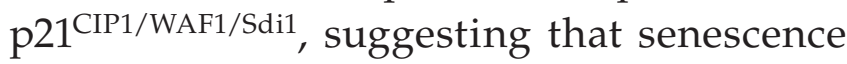
in alveolar and epithelial cells is accelerat$\mathrm{ed}^{50}$. Recent evidence from circulating endothelial progenitor cells (blood outgrowth 
endothelial cells) from smokers and patients with COPD shows increased DNA doublestrand breaks associated with senescence and impaired capacity for vascular repair compared with nonsmokers ${ }^{51}$. Two non-mutually exclusive mechanisms have been proposed, as cellular senescence could contribute to pathogenesis of COPD. First, when cellular senescence occurs, cellular proliferation slows and the balance is tipped toward apoptosis and the resulting formation of emphysematous lesions ${ }^{27}$. Second, as we have suggested above, the changes in the secretome associated with SASP could contribute to the development of COPD. Senescent cells demonstrate activation of nuclear factor kappa B (NF-kB), a major transcription factor in the regulation of inflammation. Senescent cells also release increased amounts of various inflammatory cytokines, resulting in enhanced inflammation ${ }^{52}$.

\section{Cellular senescence in idiopathic pulmonary fibrosis}

There is increasing evidence that cellular senescence plays a pivotal role in the development of IPF and could be present in different aspects of the disease.

In the lung, SA $\beta G A L$ activity is present in the three cell types involved in IPF (alveolar, bronchial, mesenchymal) in both human and mouse lungs ${ }^{48}$. The senescence marker p16 is highly upregulated in fibroblasts of the fibrotic lesions and in the overlying epithelial cells in a bleomycin model of aged mice ${ }^{53}$. Similarly, p21 is greatly expressed in human bronchial cells covering the fibrotic masses, and in vitro analyses indicated transforming growth factor beta (TGF- $\beta$ ) as a key factor in the induction of $\mathrm{p} 21^{54}$. Interestingly, it has also been demonstrated that rupatadine also impedes senescence upon bleomycin-induced fibrosis and diminishes the extent of lung fibrotic damage ${ }^{55}$.

We have recently reported that IPF fibroblasts seem to respond poorly to DNA damage and also have shorter telomeres and increased expression of p21 and p16 compared to controls. Additionally, IPF fibroblasts have decreased expression of collagen and fibronectin compared to age-matched controls at basal conditions as well as after TGF- $\beta$ stimulation. On the other hand, TGF- $\beta$ stimulation shows a tendency to decrease proliferation in IPF fibroblasts compared to controls. These findings suggest a premature senescence phenotype in IPF fibroblasts, which, taken with the lower expression of collagen found in the IPF fibroblasts, leads to the hypothesis that IPF fibroblasts are acting as senescent cells accumulating in the extracellular matrix ${ }^{35}$.

\section{STEM CELL EXHAUSTION}

Stem cell exhaustion, described as a decrease in different stem cell populations, is an integral characteristic of aging ${ }^{3}$. The size of stem cell populations depends on the balance between self-renewal and cell differentiation. When the self-renewal rate is lower than the rate of differentiation, the population declines due to exhaustion ${ }^{26}$. The reduction of the reparative capacities due to stem cell exhaustion is consequently associated with the corresponding loss of function in the different organs and the characteristic findings we observe in aging. 


\section{Stem cell exhaustion in idiopathic pulmonary fibrosis}

Bone-marrow mesenchymal stem cells (B-MSC) can migrate and participate in lung repair by modulation of inflammation ${ }^{56-58}$, but senescence can alter these effects. In a study by our group ${ }^{59}$, we compared the effect of a single dose of intratracheal bleomycin in a murine model of accelerated aging on six-month-old senescence-accelerated-prone mice (SAMP) and SA-resistant mice (SAMR) with 12-monthold mice. Fourteen days after the insult, we observed a decrease in lung repair ability in SAMP after bleomycin-induced lung injury, resulting in an increase in lung fibrosis when compared to SAMR. In SAMP, these changes were associated with higher levels of TGF- $\beta$ in the lung, a higher number of fibroblast progenitor cells (or fibrocytes) in circulation, and a decrease in the ability of MSCs to respond to the soluble signals of injury. In our current study, we have observed that only the bleomycin-injured mice that were infused with B-MSCs from young donors have lower fibrosis, confirming the fact that in aging B-MSCs, the capacity of response to fibrosis is reduced.

In addition to these results, we have recently demonstrated $^{60}$ that IPF B-MSCs show signs of accelerated senescence by $\beta$ galactosidase activity staining, with shorter telomere length, increased expression of senescence marker p21, and longer doubling times. In functional studies, IPF B-MSC and their age-matched controls were less effective than B-MSC from young controls in preventing fibrotic changes observed after bleomycin-induced lung injury in mice. In addition to these results, we have also demonstrated ${ }^{60}$ that B-MSCs obtained

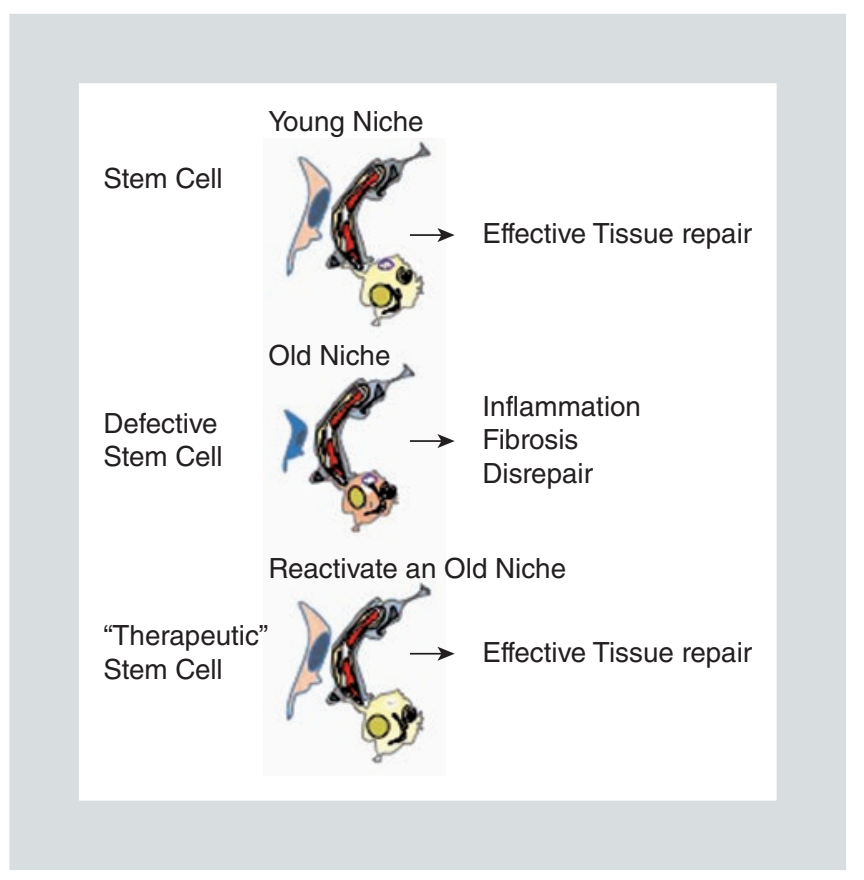

Figure 4. The role of stem cells in the lung niche.

from IPF patients are functionally defective, with changes in mitochondrial morphology and function. The IPF B-MSC mitochondria are smaller in size, exhibit decreased oxygen consumption rate, and are less glycolytic than the B-MSC from age-matched healthy individuals. These data correlate with reduced total ATP production and lower ROS generation per mitochondrion, albeit a slightly higher mitochondrial content. So, we have hypothesized that the reparative capacities of stem cells are diminished with aging. The potential therapeutic use of B-MSCs from young patients could be an option to enhance lung tissue repair (Fig. 4).

\section{CONCLUSION}

Novel mechanisms have been described in the association between aging and chronic respiratory disorders. Although COPD and IPF are different disease entities, they share 
similar abnormalities related to aging mechanisms. From all the proposed hallmarks of aging $^{3}$, we have presented novel mechanisms in telomere attrition, loss of proteostasis, mitochondrial dysfunction, cellular senescence and stem cell exhaustion. Although these mechanisms are observed in COPD and IPF, the reason why similar mechanisms lead to different diseases is unknown and warrants further research. The objective of the research into these mechanisms is to better understand these diseases and to explore novel drugs that can be used as new therapies that could improve the quality of life and survival in patients suffering from these pathologies. The link between aging and disease is still the object of fresh and innovative exploration.

\section{CONFLICT OF INTEREST}

\author{
Dr. Rojas has nothing to disclose, Dr. Sellares \\ has nothing to disclose
}

\section{REFERENCES}

1. Rojas M, Mora AL, Kapetanaki M, Weathington N, Gladwin M, Eickelberg O. Aging and Lung Disease. Clinical Impact and Cellular and Molecular Pathways. Ann Am Thorac Soc. 2015;12:S222-7.

2. Kinsella K, He W. An aging world: 2008. Washington, DC: U.S. Government Printing Office; 2009 U.S. Census Bureau International Population Reports, P95/09-1. Available at: https://niaprodfiles.s3.amazonaws.com/s3fs-public/ AgingWorld2008-web.pdf. [Accessed Jun 01, 2016].

3. López-Otín C, Blasco MA, Partridge L, Serrano M, Kroemer G. The hallmarks of aging. Cell. 2013;153:1194-217.

4. Kennedy BK, Berger SL, Brunet A, et al. Geroscience: linking aging to chronic disease. Cell. 2014;159:709-13.

5. Selman M, Buendia-Roldan I, Pardo A. Aging and pulmonary fibrosis. Rev Invest Clin. 2016;68:75-83

6. Han S, Mallampalli RK. The acute respiratory distress syndrome: from mechanism to translation. J Immunol. 2015;194:855-60.

7. Murray CJ, Lopez AD. Alternative projections of mortality and disability by cause 1990-2020: Global Burden of Disease Study. Lancet. 1997;349: 1498-504.

8. Vestbo J, Hurd SS, Agustí AG, et al. Global strategy for the diagnosis, management, and prevention of chronic obstructive pulmonary disease: GOLD executive summary. Am J Respir Crit Care Med. 2013;187:347-65.
9. Ito K, Barnes PJ. COPD as a disease of accelerated lung aging. Chest. 2009;135:173-80.

10. Ahluwalia N, Shea BS, Tager AM. New therapeutic targets in idiopathic pulmonary fibrosis: Aiming to rein in runaway wound healing responses. Am J Respir Crit Care Med. 2014;190;867-78.

11. Raghu G, Collard HR, Egan JJ, et al. An official ATS/ERS/JRS/ALAT statement: idiopathic pulmonary fibrosis: evidence-based guidelines for diagnosis and management. Am J Respir Crit Care Med. 2011;183:788-824.

12. King TE, Pardo A, Selman M. Idiopathic pulmonary fibrosis. Lancet. 2011;378:1949-61.

13. Blasco MA. Telomere length, stem cells and aging. Nat Chem Biol. 2007;3 640-9.

14. Blackburn EH, Greider CW, Szostak JW. Telomeres and telomerase: the path from maize, Tetrahymena and yeast to human cancer and aging. Nat Med. 2006;12:1133-8

15. Alder JK, Guo N, Kembou F, et al. Telomere length is a determinant of emphysema susceptibility. Am J Respir Crit Care Med. 2011;184:904-12.

16. Stanley SE, Chen JJ, Podlevsky JD, et al. Telomerase mutations in smokers with severe emphysema. J Clin Invest. 2015;125:563-70.

17. Talbert JL, Schwartz DA. Pulmonary Fibrosis, Familial. In: Pagon RA, Adam MP, Ardinger HH, Wallace SE, Amemiya A, Bean LJH, Bird TD, Fong CT, Mefford HC, Smith RJH, Stephens K, editors. GeneReviews ${ }^{\circledR}$ [Internet]. Seattle (WA): University of Washington, Seattle; 1993-2016. 2005 Jan 21 [updated 2015 Mar 19].

18. Cronkhite JT, Xing C, Raghu G, et al. Telomere shortening in familial and sporadic pulmonary fibrosis. Am J Respir Crit Care Med. 2008;178:729-37.

19. Armanios M. Telomerase and idiopathic pulmonary fibrosis. Mutat Res. 2012;730:52-8.

20. Alder JK, Chen JJ, Lancaster L, et al. Short telomeres are a risk factor for idiopathic pulmonary fibrosis. Proc Natl Acad Sci U S A. 2008;105:13051-6.

21. Armanios M. Telomeres and age-related disease: how telomere biology informs clinical paradigms. J Clin Invest. 2013;123:996-1002.

22. Stuart BD, Lee JS, Kozlitina J, et al. Effect of telomere length on survival in patients with idiopathic pulmonary fibrosis: an observational cohort study with independent validation. Lancet Respir Med. 2014;2:557-65.

23. Stuart BD, Choi J, Zaidi S, et al. Exome sequencing links mutations in PARN and RTEL1 with familial pulmonary fibrosis and telomere shortening. Nat Genet. 2015;47:512-7.

24. Cloonan SM, Choi AM. Mitochondria in lung disease. J Clin Invest. 2016, 126:809.

25. Green DR, Galluzzi L, Kroemer G. Mitochondria and the autophagy-inflammation-cell death axis in organismal aging. Science. 2011;333:1109-12.

26. Mercado N, Ito K, Barnes PJ. Accelerated ageing of the lung in COPD: new concepts. Thorax. 2015;70:482-9.

27. Faner R, Rojas M, Macnee W, Agustí A. Abnormal lung aging in chronic obstructive pulmonary disease and idiopathic pulmonary fibrosis. Am J Respir Crit Care Med. 2012;186:306-13.

28. Cloonan SM, Glass K, Laucho-Contreras ME, et al. Mitochondrial iron chelation ameliorates cigarette smoke-induced bronchitis and emphysema in mice. Nat Med. 2016;22:163-74.

29. Soulitzis N, Neofytou E, Psarrou M, et al. Downregulation of lung mitochondrial prohibitin in COPD. Respir Med. 2012;106:954-61.

30. Hoffmann RF, Zarrintan S, Brandenburg SM, et al. Prolonged cigarette smoke exposure alters mitochondrial structure and function in airway epithelial cells. Respir Res. 2013;14:97.

31. Mizumura K, Cloonan SM, Nakahira K, et al. Mitophagy-dependent necroptosis contributes to the pathogenesis of COPD. J Clin Invest. 2014 124:3987-4003.

32. Monick MM, Powers LS, Walters K, et al. Identification of an autophagy defect in smokers' alveolar macrophages. J Immunol. 2010;185:5425-35.

33. Rahman I, Skwarska E, Henry M, et al. Systemic and pulmonary oxidative stress in idiopathic pulmonary fibrosis. Free Radic Biol Med. 1999;27:60-8.

34. Bueno M, Lai YC, Romero Y, et al. PINK1 deficiency impairs mitochondrial homeostasis and promotes lung fibrosis. J Clin Invest. 2015;125:521-38. 
35. Alvarez D. IPF Fibroblasts: From The Aberrantly Activated State To The Senescent Phenotype. Am J Respir Crit Care Med. 2016;193:A2389.

36. Labbadia J, Morimoto RI. The biology of proteostasis in aging and disease. Annu Rev Biochem. 2015;84:435-64.

37. Koga H, Kaushik S, Cuervo AM. Protein homeostasis and aging: The importance of exquisite quality control. Ageing Res Rev. 2011;10:205-15.

38. Powers ET, Morimoto RI, Dillin A, Kelly JW, Balch WE. Biological and chemical approaches to diseases of proteostasis deficiency. Annu Rev Biochem. 2009;78:959-91.

39. Kelsen SG. The Unfolded Protein Response in Chronic Obstructive Pulmonary Disease. Ann Am Thorac Soc. 2016;13(Suppl 2):S138-45.

40. Cui X, Xing J, Liu Y, et al. COPD and levels of Hsp70 (HSPA1A) and Hsp27 (HSPB1) in plasma and lymphocytes among coal workers: a case-control study. Cell Stress Chaperones. 2015;20:473-81.

41. Hodge G, Roscioli E, Jersmann H, et al. Steroid resistance in COPD is associated with impaired molecular chaperone $\mathrm{Hsp} 90$ expression by pro-inflammatory lymphocytes. Respir Res. 2016;17:135.

42. Chen ZH, Kim HP, Sciurba FC, et al. Egr-1 regulates autophagy in cigarette smoke-induced chronic obstructive pulmonary disease. PLoS One. 2008;3: e3316.

43. Fujii S, Hara H, Araya J, et al. Insufficient autophagy promotes bronchial epithelial cell senescence in chronic obstructive pulmonary disease. Oncoimmunology. 2012;1:630-41.

44. Patel AS, Lin L, Geyer A, et al. Autophagy in idiopathic pulmonary fibrosis. PLoS One. 2012;7:e41394.

45. Romero Y, Bueno M, Ramirez R, et al. mTORC1 activation decreases autophagy in aging and idiopathic pulmonary fibrosis and contributes to apoptosis resistance in IPF fibroblasts. Aging Cell. 2016. [Epub ahead of print].

46. Collado M, Blasco MA, Serrano M. Cellular senescence in cancer and aging. Cell. 2007;130:223-33.

47. Rodier F, Campisi J. Four faces of cellular senescence. J Cell Biol. 2011;192: 547-56.

48. Muñoz-Espín D, Serrano M. Cellular senescence: from physiology to pathology. Nat Rev Mol Cell Biol. 2014;15:482-96.
49. Chilosi M, Carloni A, Rossi A, Poletti V. Premature lung aging and cellular senescence in the pathogenesis of idiopathic pulmonary fibrosis and COPD/emphysema. Transl Res. 2013;162:156-73.

50. Tsuji T, Aoshiba K, Nagai A. Alveolar cell senescence in patients with pulmonary emphysema. Am J Respir Crit Care. 2006;174:886-93.

51. Paschalaki KE, Starke RD, Hu Y, et al. Dysfunction of endothelial progenitor cells from smokers and chronic obstructive pulmonary disease patients due to increased DNA damage and senescence. Stem Cells. 2013;31:2813-26.

52. Shelton DN, Chang E, Whittier PS, Choi D, Funk WD. Microarray analysis of replicative senescence. Curr Biol. 1999;9:939-45.

53. Hecker L, Logsdon NJ, Kurundkar D, et al. Reversal of persistent fibrosis in aging by targeting Nox4-Nrf2 redox imbalance. Sci Transl Med. 2014 6:231ra47.

54. Minagawa S, Araya J, Numata T, et al. Accelerated epithelial cell senescence in IPF and the inhibitory role of SIRT6 in TGF- $\beta$-induced senescence of human bronchial epithelial cells. Am J Physiol Lung Cell Mol Physiol. 2011;300:L391-401.

55. Lv XX, Wang XX, Li K, et al. Rupatadine protects against pulmonary fibrosis by attenuating PAF-mediated senescence in rodents. PLoS One. 2013;8:e68631.

56. Ortiz LA, Gambelli F, McBride C, et al. Mesenchymal stem cell engraftment in lung is enhanced in response to bleomycin exposure and ameliorates its fibrotic effects. Proc Natl Acad Sci U S A. 2003;100:8407-11.

57. Rojas $\mathrm{M}, \mathrm{Xu}$ J, Woods $\mathrm{CR}$, et al. Bone marrow-derived mesenchymal stem cells in repair of the injured lung. Am J Respir Cell Mol Biol. 2005;33:145-52.

58. Zhang F, Zhang L, Jiang H-S, et al. Mobilization of bone marrow cells by CSF3 protects mice from bleomycin-induced lung injury. Respiration. 2011 82:358-68.

59. Xu J, Gonzalez ET, Iyer SS, et al. Use of senescence-accelerated mouse model in bleomycin-induced lung injury suggests that bone marrow-derived cells can alter the outcome of lung injury in aged mice. J Gerontol A Biol Sci Med Sci. 2009;64:731-9.

60. Cardenes N. Deficiencies In Mesenchymal Stem Cells From idiopathic Pulmonary Fibrosis Patients Result In Lower Capacity To Protect The Lung From injury. Am J Respir Crit Care Med. 2016;193:A3084. 